\title{
Certain transformations for multiple hypergeometric functions
}

\author{
Chuanan Wei ${ }^{1}$, Xiaoxia Wang ${ }^{2^{*}}$ and Yaqiong $\mathrm{Li}^{2}$
}

"Correspondence: xwang913@126.com

2Department of Mathematics, Shanghai University, Shanghai, 200444, P.R. China

Full list of author information is

available at the end of the article

\begin{abstract}
The aim of this research is to provide many new hypergeometric transformations of Kampé de Fériet's double hypergeometric functions and Srivastava's triple hypergeometric functions, which are obtained with the help of the relations of Appell, Humbert, Horn and Srivastava's multiple hypergeometric functions. The results are derived by the beta integral method which has been recently introduced and studied systematically by Krattenthaler and Rao.
\end{abstract}

MSC: 33C20; 33C05; 33B20

Keywords: Appell functions; Srivastava functions; multiple hypergeometric series; beta integral

\section{Introduction}

The generalized hypergeometric function ${ }_{p} F_{q}$ with $p$ numerator and $q$ denominator parameters may be defined by [1]

$$
\begin{aligned}
{ }_{p} F_{q}\left[\begin{array}{c}
\alpha_{1}, \ldots, \alpha_{p} ; \\
\beta_{1}, \ldots, \beta_{q} ;
\end{array}\right] & ={ }_{p} F_{q}\left[\alpha_{1}, \ldots, \alpha_{p} ; \beta_{1}, \ldots, \beta_{q} ; z\right] \\
& =\sum_{n \geq 0} \frac{\left(\alpha_{1}\right)_{n} \cdots\left(\alpha_{p}\right)_{n}}{\left(\beta_{1}\right)_{n} \cdots\left(\beta_{q}\right)_{n}} \cdot \frac{z^{n}}{n !}
\end{aligned}
$$

where $(\alpha)_{n}$ denotes the Pochhammer symbol (or the shifted factorial, since $(1)_{n}=n$ !) defined, for any complex number $\alpha$, by

$$
(\alpha)_{n}=\frac{\Gamma(\alpha+n)}{\Gamma(\alpha)}= \begin{cases}\alpha(\alpha+1) \cdots(\alpha+n-1), & n \in \mathbb{N}=1,2,3, \ldots, \\ 1, & n=0,\end{cases}
$$

where $\Gamma$ is the well-known gamma function. For convergence condition and properties of ${ }_{p} F_{q}$, we refer to [1, 2]. The ${ }_{p} F_{q}$ function extends the Gauss hypergeometric function

$$
{ }_{2} F_{1}\left[\begin{array}{cc}
A, B ; & z \\
C ; &
\end{array}\right]=\sum_{n \geq 0} \frac{(A)_{n}(B)_{n}}{(C)_{n}} \frac{z^{n}}{n !}
$$

by increasing the number of parameters in the numerator as well as in the denominator.

On the other hand, the notation defined and introduced by Kampé de Fériet for his double hypergeometric function of superior order was subsequently abbreviated by Burchnall

\section{Springer}

○2013 Wei et al.; licensee Springer. This is an Open Access article distributed under the terms of the Creative Commons Attribution License (http://creativecommons.org/licenses/by/2.0), which permits unrestricted use, distribution, and reproduction in any medium, provided the original work is properly cited. 
and Chaundy [3]. We, however, recall here the definition of a more general (than the one defined by Kampé de Fériet) double hypergeometric function in a slightly modified notation given by Srivastava and Panda [4, p.423, Eq. (26)]. For this, let $\left(H_{h}\right)$ denote the sequence of parameters $\left(H_{1}, H_{2}, \ldots, H_{h}\right)$, and let nonnegative integers define the Pochhammer symbols $\left(\left(H_{h}\right)\right)_{n}=\left(H_{1}\right)_{n}\left(H_{2}\right)_{n} \cdots\left(H_{h}\right)_{n}$, where, when $n=0$, the product is understood to reduce to unity. Therefore, the convenient generalization of the Kampé de Fériet function is defined as follows:

$$
F_{g: c ; d}^{h: a ; b}\left[\begin{array}{l}
\left(H_{h}\right):\left(A_{a}\right) ;\left(B_{b}\right) ; \\
\left(G_{g}\right):\left(C_{c}\right) ;\left(D_{d}\right) ;
\end{array} \quad x, y\right]=\sum_{m, n \geq 0} \frac{\left(\left(H_{h}\right)\right)_{m+n}\left(\left(A_{a}\right)\right)_{m}\left(\left(B_{b}\right)\right)_{n}}{\left(\left(G_{g}\right)\right)_{m+n}\left(\left(C_{c}\right)\right)_{m}\left(\left(D_{d}\right)\right)_{n}} \frac{x^{m}}{m !} \frac{y^{n}}{n !} .
$$

For more details about the convergence of this function, we refer to [4]. Various authors (see, e.g., [5-9]) have discussed the reducibility of the Kampé de Fériet function.

Finally, we introduce Srivastava's $[10, \mathrm{p} .44]$ triple hypergeometric series $F^{(3)}[x, y, z]$ defined by

$$
\begin{aligned}
F^{(3)}[x, y, z] & \equiv F^{(3)}\left[\begin{array}{l}
(a)::(b) ;\left(b^{\prime}\right) ;\left(b^{\prime \prime}\right):(c) ;\left(c^{\prime}\right) ;\left(c^{\prime \prime}\right) ; \\
(e)::(g) ;\left(g^{\prime}\right) ;\left(g^{\prime \prime}\right):(h) ;\left(h^{\prime}\right) ;\left(h^{\prime \prime}\right) ;
\end{array} \quad x, y, z\right] \\
& =\sum_{m, n, p=0}^{\infty} \Lambda(m, n, p) \frac{x^{m}}{m !} \frac{y^{n}}{n !} \frac{z^{p}}{p !},
\end{aligned}
$$

where, for convenience,

$$
\begin{aligned}
\Lambda(m, n, p)= & \frac{\prod_{j=1}^{A}\left(a_{j}\right)_{m+n+p} \prod_{j=1}^{B}\left(b_{j}\right)_{m+n} \prod_{j=1}^{B^{\prime}}\left(b_{j}^{\prime}\right)_{n+p} \prod_{j=1}^{B^{\prime \prime}}\left(b_{j}^{\prime \prime}\right)_{m+p}}{\prod_{j=1}^{E}\left(e_{j}\right)_{m+n+p} \prod_{j=1}^{G}\left(g_{j}\right)_{m+n} \prod_{j=1}^{G^{\prime}}\left(g_{j}^{\prime}\right)_{n+p} \prod_{j=1}^{G^{\prime \prime}}\left(g_{j}^{\prime \prime}\right)_{m+p}} \\
& \cdot \frac{\prod_{j=1}^{C}\left(c_{j}\right)_{m} \prod_{j=1}^{C^{\prime}}\left(c_{j}^{\prime}\right)_{n} \prod_{j=1}^{C^{\prime \prime}}\left(c_{j}^{\prime \prime}\right)_{p}}{\prod_{j=1}^{H}\left(h_{j}\right)_{m} \prod_{j=1}^{H^{\prime}}\left(h_{j}^{\prime}\right)_{n} \prod_{j=1}^{H^{\prime \prime}}\left(h_{j}^{\prime \prime}\right)_{p}}
\end{aligned}
$$

and $(a)$ abbreviates the array of $A$ parameters $a_{1}, \ldots, a_{A}$, with similar interpretations for $(b),\left(b^{\prime}\right),\left(b^{\prime \prime}\right)$, and so on.

The reduction formulas of the general hypergeometric functions in one, two and more variables have been investigated, and some of them can be seen in $[5,10]$.

In addition, the beta function $\mathrm{B}(\alpha, \beta)$ is defined by the first integral and known to be evaluated as the second one as follows:

$$
\mathrm{B}(\alpha, \beta)= \begin{cases}\int_{0}^{1} t^{\alpha-1}(1-t)^{\beta-1} d t & (\operatorname{Re}(\alpha)>0 ; \operatorname{Re}(\beta)>0) ; \\ \frac{\Gamma(\alpha) \Gamma(\beta)}{\Gamma(\alpha+\beta)} & \left(\alpha, \beta \in \mathbb{C} \backslash \mathbb{Z}_{0}^{-}\right) .\end{cases}
$$

Recently, Krattenthaler and Rao [11] (see also [12]) made a systematic use of the so-called beta integral method, a method of deriving new hypergeometric identities from old ones by mainly using the beta integral in (1.3) based on the Mathematica Package HYP, to illustrate several interesting identities for the hypergeometric functions and Kampé de Fériet functions.

There are many relations of Appell, Humbert, Horn and Srivastava's multiple hypergeometric functions [13-16] from which we can establish many new transformations of 
Kampé de Fériet's double hypergeometric function and Srivastava's triple hypergeometric functions by employing the so-called beta integral method. Moreover, in our work, we use the beta integral to establish transformations two and three times, and this is a generalization of Krattenthaler and Rao's method.

\section{The new transformations from Appell functions}

The following four functions are Appell functions $[17,18]$ which are famous in the field of the hypergeometric functions $[6,10,15]$ :

$$
\begin{aligned}
& F_{1}\left[a ; b_{1}, b_{2} ; c ; x, y\right]:=\sum_{m, n \geq 0} \frac{(a)_{m+n}\left(b_{1}\right)_{m}\left(b_{2}\right)_{n}}{(c)_{m+n}} \frac{x^{m}}{m !} \frac{y^{n}}{n !} ; \\
& F_{2}\left[a ; b_{1}, b_{2} ; c_{1}, c_{2} ; x, y\right]:=\sum_{m, n \geq 0} \frac{(a)_{m+n}\left(b_{1}\right)_{m}\left(b_{2}\right)_{n}}{\left(c_{1}\right)_{m}\left(c_{2}\right)_{n}} \frac{x^{m}}{m !} \frac{y^{n}}{n !} ; \\
& F_{3}\left[a_{1}, a_{2} ; b_{1}, b_{2} ; c ; x, y\right]:=\sum_{m, n \geq 0} \frac{\left(a_{1}\right)_{m}\left(a_{2}\right)_{n}\left(b_{1}\right)_{m}\left(b_{2}\right)_{n}}{(c)_{m+n}} \frac{x^{m}}{m !} \frac{y^{n}}{n !} ; \\
& F_{4}\left[a ; b ; c_{1}, c_{2} ; x, y\right]:=\sum_{m, n \geq 0} \frac{(a)_{m+n}(b)_{m+n}}{\left(c_{1}\right)_{m}\left(c_{2}\right)_{n}} \frac{x^{m}}{m !} \frac{y^{n}}{n !} .
\end{aligned}
$$

In this part, we present the transformations which are obtained from Appell's four hypergeometric functions and we just take two of them as examples and the others can be derived in the same manner.

We give two theorems as examples in this part.

Theorem 1 Assuming $b_{1}$ or $b_{2}$ is a nonpositive integer, and $d$ and e are not nonpositive integers, the following result holds true:

$$
\begin{aligned}
F_{2: 0 ; 0}^{1: 2 ; 2}\left[\begin{array}{cc}
a: b_{1}, e+b_{2} ; b_{2}, d+b_{1}-e ; & 1,1 \\
c, d+b_{1}+b_{2}:-;-;
\end{array}\right] \\
=\Gamma\left[\begin{array}{l}
e, d-e, d+b_{1}+b_{2} \\
d, e+b_{2}, d+b_{1}-e
\end{array} \sum_{m, n \geq 0} \frac{(c-a)_{m+n}(e)_{m-n}\left(b_{1}\right)_{m}\left(b_{2}\right)_{n}}{(c)_{m+n}(1+e-d)_{m-n} m ! n !} .\right.
\end{aligned}
$$

It is interesting that the series on the right-hand side of the assertion of Theorem 1 is similar to Horn's $G_{1}$ and $H_{1}$ [13, pp.224-225].

Proof We start with the following well-known transformation of Appell function $F_{1}[15$, p.217, Eq. (8.3.2)]:

$$
F_{1}\left[a ; b_{1}, b_{2} ; c ; x, y\right]=(1-x)^{-b_{1}}(1-y)^{-b_{2}} F_{1}\left[c-a ; b_{1}, b_{2} ; c ; \frac{x}{x-1}, \frac{y}{y-1}\right] .
$$

Setting $y=1-x$ in (2.1), we get the following identity of $x$ :

$$
F_{1}\left[a ; b_{1}, b_{2} ; c ; x, 1-x\right]=(1-x)^{-b_{1}} x^{-b_{2}} F_{1}\left[c-a ; b_{1}, b_{2} ; c ; \frac{x}{x-1}, \frac{1-x}{-x}\right] .
$$

Now, multiplying both sides of equation $(2.2)$ by $x^{b_{2}+e-1}(1-x)^{b_{1}+d-e-1}$, integrating the resulting equation with respect to $x$ from 0 to 1 , expressing the involved $F_{1}$ as series, in- 
terchanging the order of integration and summation, which is easily seen to be justified due to the uniform convergence of the series in the interval $(0,1)$ involved in the process, evaluating the integral on both sides using the beta integral (1.3), then after some simplification, summing up the series on both sides, we easily arrive at the assertion of Theorem 1.

Theorem 2 Assuming $b_{1}$ or $b_{2}$ is a nonpositive integer, and $d_{1}, d_{2}, e_{1}$ and $e_{2}$ are not nonpositive integers, the following result holds true:

$$
\begin{aligned}
& F_{1: 1 ; 1}^{1: 2 ; 2}\left[\begin{array}{cc}
a: b_{1}, e_{1} ; b_{2}, e_{2} ; & 1,1 \\
c: b_{1}+d_{1} ; b_{2}+d_{2} ; &
\end{array}\right] \\
& =\Gamma\left[\begin{array}{l}
d_{1}-e_{1}, d_{2}-e_{2}, b_{1}+d_{1}, b_{2}+d_{2} \\
d_{1}, d_{2}, b_{1}+d_{1}-e_{1}, b_{2}+d_{2}-e_{2}
\end{array}\right] \\
& \cdot F_{1: 1 ; 1}^{1: 2 ; 2}\left[\begin{array}{cc}
c-a: b_{1}, e_{1} ; b_{2}, e_{2} ; & 1,1 \\
c: 1+e_{1}-d_{1} ; 1+e_{2}-d_{2} ; &
\end{array}\right] \text {. }
\end{aligned}
$$

Proof We prove Theorem 2 by applying the beta integral method twice. Now, multiplying both sides of equation (2.1) by $x^{e_{1}-1}(1-x)^{b_{1}+d_{1}-e_{1}-1} y^{e_{2}-1}(1-x)^{b_{2}+d_{2}-e_{2}-1}$, integrating the resulting equation with respect to $x$ and $y$ both from 0 to 1 , expressing the involved $F_{1}$ as series, interchanging the order of integration and summation, which is easily seen to be justified due to the uniform convergence of the series in the interval $(0,1)$ involved in the process, evaluating the integral on both sides using the beta integral (1.3) twice, then after some simplification, summing up the series on both sides, we easily arrive at the assertion of Theorem 2.

In the following part, all the transformations are obtained by the beta integral method and the derivations are the same as in the proof of Theorems 1 and 2. We will just present the results without their proofs.

If we start with the formula [16, Eq. (8)] with $y=x$,

$$
F_{4}[a ; b ; c, 1+a+b-c ; x(1-x), x(1-x)]={ }_{2} F_{1}\left[\begin{array}{cc}
a, b ; & x \\
c ; &
\end{array}\right]{ }_{2} F_{1}\left[\begin{array}{cc}
a, b ; & x \\
1+a+b-c ; & x
\end{array}\right]
$$

we have the following transformation:

$$
F_{2: 1 ; 1 ;}^{4: 0 ; 0}\left[\begin{array}{cc}
a, b, e, d-e:-;-; & \frac{1}{4}, \frac{1}{4} \\
\frac{d}{2}, \frac{d+1}{2}: c ; 1+a+b-c ;
\end{array}\right]=F_{1: 1 ; 1}^{1: 2 ; 2}\left[\begin{array}{cc}
e: a, b ; a, b ; & 1,1 \\
d: c ; 1+a+b-c ; &
\end{array}\right]
$$

with $e$ being a nonpositive integer.

We start with the formula [16, Eq. (8)] with $y=1-x$,

$$
F_{4}\left[a ; b ; c, 1+a+b-c ; x^{2},(1-x)^{2}\right]={ }_{2} F_{1}\left[\begin{array}{cc}
a, b ; & x \\
c ; &
\end{array}\right]{ }_{2} F_{1}\left[\begin{array}{cc}
a, b ; & 1-x \\
1+a+b-c ; &
\end{array}\right],
$$


we have

$$
F_{2: 1 ; 1}^{2: 2 ; 2}\left[\begin{array}{ll}
a, b: \frac{e}{2}, \frac{e+1}{2} ; \frac{d-e}{2}, \frac{d-e+1}{2} ; \\
\frac{d}{2}, \frac{d+1}{2}: c ; 1+a+b-c ;
\end{array} \quad 1,1\right]=F_{1: 1 ; 1}^{0: 3 ; 3}\left[\begin{array}{cc}
-: a, b, e ; a, b, d-e ; & 1,1 \\
d: c ; 1+a+b-c ; &
\end{array}\right],
$$

where, $a, b, e$ or $d-e$ is a nonpositive integer.

Especially, the right-hand side of (2.4) is equal to the left-hand side of the result [11, Eq. (4.5)] by Krattenthaler and Rao:

$$
F_{1: 1 ; 1}^{1: 2 ; 2}\left[\begin{array}{cc}
d: a, b ; a, b ; & 1,1 \\
e: 1+a+b-c ; c ; &
\end{array}\right]={ }_{6} F_{5}\left[\begin{array}{cc}
a, \frac{a+b}{2}, \frac{1+a+b}{2}, b, d, e-d ; & \\
a+b, 1+a+b-c, c, \frac{e}{2}, \frac{e+1}{2} ; & 1
\end{array}\right] .
$$

Comparing the identities (2.4) with (2.5), we have a new relation between the double hypergeometric function and the generalized hypergeometric function ${ }_{6} F_{5}$ :

$$
F_{2: 1 ; 1}^{4: 0 ; 0}\left[\begin{array}{cc}
a, b, e, d-e:-;-; & \frac{1}{4}, \frac{1}{4} \\
\frac{d}{2}, \frac{d+1}{2}: c ; 1+a+b-c ;
\end{array}\right]={ }_{6} F_{5}\left[\begin{array}{cc}
a, \frac{a+b}{2}, \frac{1+a+b}{2}, b, e, d-e ; & \\
a+b, 1+a+b-c, c, \frac{d}{2}, \frac{d+1}{2} ; & 1
\end{array}\right] .
$$

If we start with the transformation [16, Eq. (8)],

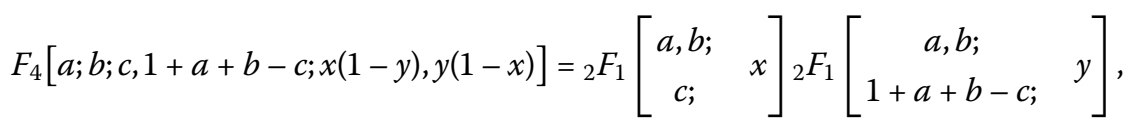

applying the beta integral method twice, after some simplification, we can get the following transformation:

$$
F_{2: 1 ; 1}^{2: 2 ; 2}\left[\begin{array}{cc}
a, b: e_{1}, d_{2}-e_{2} ; d_{1}-e_{1}, e_{2} ; & 1,1 \\
d_{1}, d_{2}: c ; 1+a+b-c ; &
\end{array}\right]=F_{0: 2 ; 2}^{0: 3 ; 3}\left[\begin{array}{cc}
-: a, b, e_{1} ; a, b, e_{2} ; & 1,1 \\
-: c, d_{1} ; 1+a+b-c, d_{2} ; &
\end{array}\right]
$$

where $a$ is a nonpositive integer.

If we start with the transformation [16, Eq. (32)] with $x \rightarrow u x$,

$$
F_{2}\left[a ; b_{1}, b_{2} ; c_{1}, c_{2} ; u x, 1\right]=\Gamma\left[\begin{array}{c}
c_{2}, c_{2}-a-b_{2} \\
c_{2}-a, c_{2}-b_{2}
\end{array}\right]{ }_{3} F_{2}\left[\begin{array}{c}
a, b_{1}, 1+a-c_{2} ; \\
c_{1}, 1+a+b_{2}-c_{2} ;
\end{array}\right] \text { ux }
$$

we have

$$
F_{0: 2 ; 1}^{1: 2 ; 1}\left[\begin{array}{ll}
a: b_{1}, e ; b_{2} ; & u, 1 \\
-: c_{1}, d ; c_{2} ; &
\end{array}\right]=\Gamma\left[\begin{array}{l}
c_{2}, c_{2}-a-b_{2} \\
c_{2}-a, c_{2}-b_{2}
\end{array}\right]{ }_{4} F_{3}\left[\begin{array}{c}
a, b_{1}, 1+a-c_{2}, e ; \\
c_{1}, 1+a+b_{2}-c_{2}, d ;
\end{array}\right]
$$

In fact, this result can also be derived by the following Gauss summation theorem $[2, \$ 1.3]$

$$
{ }_{2} F_{1}\left[\begin{array}{cc}
a, b ; & 1 \\
c ; & 1
\end{array}\right]=\Gamma\left[\begin{array}{c}
c, c-a-b \\
c-a, c-b
\end{array}\right]
$$

and the definition of the double hypergeometric function $F_{2}$ directly. 
If we start with the equation [16, Eq. (54)] with $y=-x$,

$$
\begin{aligned}
F_{4} & {\left[a ; b ; c, a-b+1 ; x, x^{2}\right] } \\
& =(1-x)^{-2 a} F_{2}\left[a ; b, a-b+\frac{1}{2} ; c, 2 a-2 b+1 ; \frac{x}{(1-x)^{2}}, \frac{-4 x}{(1-x)^{2}}\right],
\end{aligned}
$$

we get the following transformation:

$$
\begin{aligned}
& F_{2: 1 ; 1}^{3: 1 ; 1}\left[\begin{array}{cc}
a, e, 1-d: b ; a-b+\frac{1}{2} ; & -\frac{1}{4}, 1 \\
\frac{1+e-d}{2}, 1+\frac{e-d}{2}: c ; 2 a-2 b+1 ; &
\end{array}\right] \\
& =\Gamma\left[\begin{array}{l}
d, d+2 a-e \\
d+2 a, d-e
\end{array}\right] \sum_{m, n \geq 0} \frac{(a)_{m+n}(b)_{m+n}(e)_{m+2 n}}{(c)_{m}(a-b+1)_{n}(d+2 a)_{m+2 n} m ! n !},
\end{aligned}
$$

and the double series on the left-hand side of the above identity can be considered as a generalization of Horn's $H_{3}$ [13].

If we start with the transformation [16, Eq. (54)] with $y \rightarrow-y$,

$$
\begin{aligned}
F_{4} & {\left[a ; b ; c, a-b+1 ; x, y^{2}\right] } \\
& =(1-y)^{-2 a} F_{2}\left[a ; b, a-b+\frac{1}{2} ; c, 2 a-2 b+1 ; \frac{x}{(1-y)^{2}}, \frac{-4 y}{(1-y)^{2}}\right],
\end{aligned}
$$

we have, after applying the beta integral twice,

$$
\begin{aligned}
\left.F_{0: 2 ; 3}^{2: 1 ; 2}\left[\begin{array}{c}
a, b: e_{1} ; \frac{e_{2}}{2}, \frac{e_{2}+1}{2} ; \\
-: c, d_{1} ; a-b+1, \frac{d_{2}+2 a}{2}, \frac{d_{2}+2 a+1}{2} ;
\end{array}\right] 1,1\right] \\
=\Gamma\left[\begin{array}{l}
d_{2}-e_{2}, d_{2}+2 a \\
d_{2}, d_{2}+2 a-e_{2}
\end{array}\right] \\
\cdot \sum_{m, n \geq 0} \frac{(a)_{m+n}(b)_{m}\left(e_{1}\right)_{m}\left(a-b+\frac{1}{2}\right)_{n}\left(e_{2}\right)_{n}\left(1-d_{2}\right)_{2 m+n}}{\left(\frac{1+e_{2}-d_{2}}{2}\right)_{m+n}\left(1+\frac{e_{2}-d_{2}}{2}\right)_{m+n}(c)_{m}\left(d_{1}\right)_{m}(2 a-2 b+1)_{n}} \frac{\left(\frac{1}{4}\right)^{m}}{m ! n !} .
\end{aligned}
$$

The double hypergeometric series on the right-hand side of the above identity can be considered as a generalization of Horn's $H_{4}$ [13] and is similar to Exton's $X_{8}$ [19].

Substituting $y$ by $1-x$ in the result [16, Eq. (55)], we have

$$
F_{4}\left[\frac{a}{2} ; \frac{a+1}{2} ; b_{1}+\frac{1}{2}, b_{2}+\frac{1}{2} ; x^{2},(1-x)^{2}\right]=2^{-a} F_{2}\left[a ; b_{1}, b_{2} ; 2 b_{1}, 2 b_{2} ; x, 1-x\right] .
$$

We then obtain the following transformation by the beta integral method:

$$
F_{2: 1 ; 1 ;}^{2: 2 ; 2}\left[\begin{array}{c}
\frac{a}{2}, \frac{a+1}{2}: \frac{e}{2}, \frac{e+1}{2} ; \frac{d-e}{2}, \frac{d-e+1}{2} ; \quad 1,1 \\
\frac{d}{2}, \frac{d+1}{2}: b_{1}+\frac{1}{2} ; b_{2}+\frac{1}{2} ;
\end{array}\right]=2^{-a} F_{1: 1 ; 1}^{1: 2 ; 2}\left[\begin{array}{cc}
a: b_{1}, e ; b_{2}, d-e ; & 1,1 \\
d: 2 b_{1} ; 2 b_{2} ; &
\end{array}\right],
$$

where $a$ is a nonpositive integer.

If we start with the equation [16, Eq. (77)] with $x \rightarrow u x$,

$$
F_{1}\left[a ; b_{1}, b_{2} ; c ; u x, u x\right]={ }_{2} F_{1}\left[\begin{array}{cc}
a, b_{1}+b_{2} ; & u x \\
c ; &
\end{array}\right],
$$


and assume that $a$ is a nonpositive integer, we have

$$
F_{2: 0 ; 0}^{2: 1 ; 1}\left[\begin{array}{cc}
a, e: b_{1} ; b_{2} ; \\
c, d:-;-; & u, u
\end{array}\right]={ }_{3} F_{2}\left[\begin{array}{c}
a, b_{1}+b_{2}, e ; \\
c, d ;
\end{array}\right]
$$

If we start with the formula [16, Eq. (102)] (see also [15, Eq. (8.3.8)]) with $y=x$,

$$
F_{2}\left[a ; b_{1}, b_{2} ; c_{1}, c_{2} ; x, x\right]=(1-x)^{-a} F_{2}\left[a ; c_{1}-b_{1}, b_{2} ; c_{1}, c_{2} ; \frac{x}{x-1}, \frac{x}{1-x}\right]
$$

and provided $a$ is a nonpositive integer, we have

$$
F_{1: 1 ; 1}^{2: 1 ; 1}\left[\begin{array}{cc}
a, e: b_{1} ; b_{2} ; \\
a+d: c_{1} ; c_{2} ; & 1,1
\end{array}\right]=\Gamma\left[\begin{array}{c}
d-e, a+d \\
d, a+d-e
\end{array}\right] F_{1: 1 ; 1}^{2: 1 ; 1}\left[\begin{array}{cc}
a, e: c_{1}-b_{1} ; b_{2} ; & 1,-1 \\
1+e-d: c_{1} ; c_{2} ; &
\end{array}\right]
$$

If we start with the formula [16, Eq. (104)] (see also [15, Eq. (8.3.10)]) with $y \rightarrow-u x$ and $x \rightarrow u x$

$$
F_{2}\left[a ; b_{1}, b_{2} ; c_{1}, c_{2} ; u x,-u x\right]=F_{2}\left[a ; c_{1}-b_{1}, c_{2}-b_{2} ; c_{1}, c_{2} ;-u x, u x\right]
$$

and assume that $a$ is a nonpositive integer, we obtain the following identity:

$$
F_{1: 1 ; 1}^{2: 1 ; 1}\left[\begin{array}{cc}
a, e: b_{1} ; b_{2} ; & u,-u \\
d: c_{1} ; c_{2} ; &
\end{array}\right]=F_{1: 1 ; 1}^{2: 1 ; 1}\left[\begin{array}{cc}
a, e: c_{1}-b_{1} ; c_{2}-b_{2} ; & -u, u \\
d: c_{1} ; c_{2} ; &
\end{array}\right]
$$

If we start with the relation [16, Eq. (124)] with $x \rightarrow u x$,

$$
F_{2}\left[a ; b_{1}, b_{2} ; 2 b_{1}, 2 b_{2} ; u x,-u x\right]={ }_{4} F_{3}\left[\begin{array}{c}
\frac{a}{2}, \frac{a+1}{2}, \frac{b_{1}+b_{2}}{2}, \frac{b_{1}+b_{2}+1}{2} ; \\
b_{1}+\frac{1}{2}, b_{2}+\frac{1}{2}, b_{1}+b_{2} ;
\end{array} \quad u^{2} x^{2}\right]
$$

we obtain the following transformation by using the beta integral method on $x$ :

$$
F_{1: 1 ; 1}^{2: 1 ; 1}\left[\begin{array}{ll}
a, e: b_{1} ; b_{2} ; \\
d: 2 b_{1} ; 2 b_{2} ;
\end{array} \quad u,-u\right]={ }_{6} F_{5}\left[\begin{array}{c}
\frac{a}{2}, \frac{a+1}{2}, \frac{b_{1}+b_{2}}{2}, \frac{b_{1}+b_{2}+1}{2}, \frac{e}{2}, \frac{e+1}{2} ; \\
b_{1}+\frac{1}{2}, b_{2}+\frac{1}{2}, b_{1}+b_{2}, \frac{d}{2}, \frac{d+1}{2} ; \quad u^{2}
\end{array}\right]
$$

where $a$ is a nonpositive integer.

Remark In fact, there are many other identities of Appell functions which can be used to establish the new transformations. Here we just illustrate three results.

\section{The transformations from Horn's functions $G_{1}$ and $G_{2}$}

We first present the definitions of Horn's double hypergeometric functions $[13,18]$ as follows:

$$
\begin{aligned}
& G_{1}\left(\alpha ; \beta_{1}, \beta_{2} ; x, y\right)=\sum_{m, n \geq 0} \frac{(\alpha)_{m+n}\left(\beta_{1}\right)_{n-m}\left(\beta_{2}\right)_{m-n}}{m ! n !} x^{m} y^{n} \quad(|x|+|y|<1), \\
& G_{2}\left(\alpha_{1}, \alpha_{2} ; \beta_{1}, \beta_{2} ; x, y\right)=\sum_{m, n \geq 0} \frac{\left(\alpha_{1}\right)_{m}\left(\alpha_{2}\right)_{n}\left(\beta_{1}\right)_{n-m}\left(\beta_{2}\right)_{m-n}}{m ! n !} x^{m} y^{n} \quad(|x|,|y|<1) .
\end{aligned}
$$


If we start with the transformation between $F_{2}$ and $G_{2}$ in [20, Eq. (5.6)] (see also [21]) with $y \rightarrow-x$ and $x \rightarrow-x$,

$$
\begin{aligned}
& G_{2}\left(\alpha_{1}, \alpha_{2} ; \beta_{1}, \beta_{2} ;-x,-x\right) \\
& \quad=(1-x)^{-\alpha_{1}-\alpha_{2}} F_{2}\left[1-\beta_{1}-\beta_{2} ; \alpha_{1}, \alpha_{2} ; 1-\beta_{1}, 1-\beta_{2} ; \frac{-x}{1-x}, \frac{-x}{1-x}\right],
\end{aligned}
$$

we obtain

$$
\begin{aligned}
F_{1: 1 ; 1}^{2: 1 ; 1} & {\left[\begin{array}{cc}
e, 1-\beta_{1}-\beta_{2}: \alpha_{1} ; \alpha_{2} ; & 1,1 \\
1+e-d: 1-\beta_{1} ; 1-\beta_{2} ; &
\end{array}\right] } \\
= & \Gamma\left[\begin{array}{l}
d, \alpha_{1}+\alpha_{2}+d-e \\
d-e, \alpha_{1}+\alpha_{2}+d
\end{array}\right] \sum_{m, n \geq 0} \frac{(e)_{m+n}\left(\beta_{1}\right)_{n-m}\left(\beta_{2}\right)_{m-n}\left(\alpha_{1}\right)_{m}\left(\alpha_{2}\right)_{n}}{(-1)^{m+n}\left(\alpha_{1}+\alpha_{2}+d\right)_{m+n} m ! n !},
\end{aligned}
$$

where $e$ is a nonpositive integer. In fact, the double hypergeometric function on the righthand side of the above identity can be considered as a generalization of $G_{1}$.

If we start with the transformation between $F_{4}$ and $G_{1}$ in [20, Eq. (5.7)] (see also [21]) with $y=-2 x$,

$$
G_{1}\left(\alpha ; \beta_{1}, \beta_{2} ; x,-2 x\right)=(1-x)^{-\alpha} F_{4}\left[\alpha, 1-\beta_{1}-\beta_{2} ; 1-\beta_{1}, 1-\beta_{2} ; \frac{x}{1-x}, \frac{-2 x}{1-x}\right],
$$

and provided that $a$ or $e$ is a nonpositive integer, we have

$$
\begin{aligned}
F_{1: 1 ; 1}^{3: 000} & {\left[\begin{array}{cc}
e, \alpha, 1-\beta_{1}-\beta_{2}:-;-; & -1,2 \\
1+e-d: 1-\beta_{1} ; 1-\beta_{2} ; &
\end{array}\right] } \\
= & \Gamma\left[\begin{array}{l}
d, \alpha+d-e \\
d-e, \alpha+d
\end{array}\right] \sum_{m, n \geq 0} \frac{(e)_{m+n}(\alpha)_{m+n}\left(\beta_{1}\right)_{n-m}\left(\beta_{2}\right)_{m-n}}{(\alpha+d)_{m+n} m ! n !}(-2)^{n} .
\end{aligned}
$$

The double hypergeometric function on the right-hand side of the above identity can be considered as a generalization of $G_{1}$.

\section{The transformations from Humbert's functions $\Psi_{2}, \Phi_{2}$ and $\Phi_{3}$}

First, we present Humbert's three functions defined by (see [22, 23])

$$
\begin{aligned}
& \Phi_{3}(b ; c ; x, y)=\sum_{m, n \geq 0} \frac{(b)_{m}}{(c)_{m+n}} \frac{x^{m} y^{n}}{m ! n !}, \\
& \Psi_{2}(a ; b, c ; x, y)=\sum_{m, n \geq 0} \frac{(a)_{m+n}}{(b)_{m}(c)_{n}} \frac{x^{m} y^{n}}{m ! n !} \\
& \Phi_{2}(a, b ; c ; x, y)=\sum_{m, n \geq 0} \frac{(a)_{m}(b)_{n}}{(c)_{m+n}} \frac{x^{m} y^{n}}{m ! n !} .
\end{aligned}
$$

The above series converge absolutely for any $x, y \in \mathbb{C}$.

If we start with the transformation [14, Eq. (4)] with $x \rightarrow u x$ and $y \rightarrow-u x$,

$$
\Psi_{2}(b ; b, c ; u x,-u x)=\Phi_{3}\left(c-b ; c ; u x,-u^{2} x^{2}\right),
$$


we have

$$
F_{1: 1 ; 1}^{2: 0 ; 0}\left[\begin{array}{c}
b, e:-;-; \\
d: b ; c ;
\end{array} \quad u,-u\right]=\sum_{m, n \geq 0} \frac{(e)_{m+2 n}(c-b)_{m}}{(d)_{m+2 n}(c)_{m+n}} \frac{u^{m}\left(-u^{2}\right)^{n}}{m ! n !} .
$$

Also, the right-hand side of the above identity can be considered as a generalization of Horn's $H_{3}$ [13] and is similar to Exton's $X_{8}$ [19]. The special case of $b=c$ in the above relation can be written as

$$
F_{1: 1 ; 1}^{2: 000}\left[\begin{array}{cc}
b, e:-;-; & u,-u \\
d: b ; b ; &
\end{array}\right]={ }_{2} F_{3}\left[\begin{array}{cc}
\frac{e}{2}, \frac{e+1}{2} ; & -u^{2} \\
b, \frac{d}{2}, \frac{d+1}{2} ; &
\end{array}\right] .
$$

This identity is a relation between general and double hypergeometric functions.

If we start with the transformation [14, Eq. (4)] with $x \rightarrow u x$ and $y \rightarrow u-u x$,

$$
\Psi_{2}(b ; b, c ; u x, u-u x)=e^{u} \cdot \Phi_{3}\left(c-b ; c ; u x-u, u^{2} x(1-x)\right),
$$

we have

$$
F_{1: 1 ; 1}^{1: 1 ; 1}\left[\begin{array}{cc}
b: e ; d-e ; & u, u \\
d: b ; c ; &
\end{array}\right]=e^{u} \sum_{m, n \geq 0} \frac{(d-e)_{m+n}(c-b)_{m}(e)_{n}}{(d)_{m+2 n}(c)_{m+n}} \frac{(-u)^{m} u^{2 n}}{m ! n !} .
$$

The special case of the above identity with $b=c$ is a relation between a double hypergeometric function and a general hypergeometric function

$$
F_{1: 1 ; 1}^{1: 1 ; 1}\left[\begin{array}{cc}
b: e ; d-e ; & u, u \\
d: b ; b ; &
\end{array}\right]=e^{u} \cdot{ }_{2} F_{3}\left[\begin{array}{cc}
e, d-e ; & u^{2} \\
b, \frac{d}{2}, \frac{d+1}{2} ; & \frac{1}{4}
\end{array}\right] \text {. }
$$

If we start with the transformation $[24, \$ 7.2 .4$, Eq. (92)] with $x \rightarrow u x$,

$$
\Psi_{2}(a ; b, c ; u x, u x)={ }_{3} F_{3}\left[\begin{array}{ll}
a, \frac{c+b-1}{2}, \frac{c+b}{2} ; \\
b, c, c+b-1 ;
\end{array} \quad 4 u x\right],
$$

we have

$$
F_{1: 1 ; 1}^{2: 0 ; 0}\left[\begin{array}{cc}
a, e:-;-; & u, u \\
d: b ; c ; &
\end{array}\right]={ }_{4} F_{4}\left[\begin{array}{cc}
a, \frac{c+b-1}{2}, \frac{c+b}{2}, e ; & 4 u \\
b, c, c+b-1, d ; &
\end{array}\right] .
$$

This relation converges for every $u \in \mathbb{C}$.

If we start with the transformation [14, Eq. (8)] with $x \rightarrow u x$,

$$
\Psi_{2}(a ; c, c ; u x,-u x)={ }_{2} F_{3}\left[\begin{array}{cc}
\frac{a}{2}, \frac{a+1}{2} ; & -u^{2} x^{2} \\
c, \frac{c}{2}, \frac{c+1}{2} ; &
\end{array}\right],
$$

we have

$$
F_{1: 1 ; 1}^{2: 0 ; 0}\left[\begin{array}{cc}
a, e:-;-; & u,-u \\
d: c ; c ; &
\end{array}\right]={ }_{4} F_{5}\left[\begin{array}{cc}
\frac{a}{2}, \frac{a+1}{2}, \frac{e}{2}, \frac{e+1}{2} ; & -u^{2} \\
c, \frac{c}{2}, \frac{c+1}{2}, \frac{d}{2}, \frac{d+1}{2} ; &
\end{array}\right] .
$$


If we start with the equation [14, Eq. (9)] with $x \rightarrow u x$,

$$
\Psi_{2}\left(2 a ; 2 a+\frac{1}{2}, 2 a+\frac{1}{2} ; u x,-u x\right)={ }_{1} F_{1}\left(a ; 2 a+\frac{1}{2} ;-2 i u x\right){ }_{1} F_{1}\left(a ; 2 a+\frac{1}{2} ; 2 i u x\right)
$$

we have

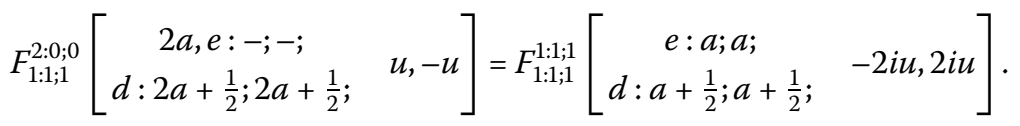

Comparing the identities (4.1) with (4.2), we can obtain a new transformation

$$
{ }_{4} F_{5}\left[\begin{array}{c}
a, a+\frac{1}{2}, \frac{e}{2}, \frac{e+1}{2} ; \\
2 a+\frac{1}{2}, a+\frac{1}{4}, a+\frac{3}{4}, \frac{d}{2}, \frac{d+1}{2} ;
\end{array} \quad-u^{2}\right]=F_{1: 1 ; 1}^{1: 1 ;[}\left[\begin{array}{cc}
e: a ; a ; & -2 i u, 2 i u \\
d: a+\frac{1}{2} ; a+\frac{1}{2} ; &
\end{array}\right] .
$$

If we start with the formula [14, Eq. (10)] with $x \rightarrow u x$,

$$
\Phi_{2}(a, b ; c ; u x, u x)={ }_{1} F_{1}(a+b ; c ; u x)
$$

we have

$$
F_{2: 0 ; 0}^{1: 1 ; 1}\left[\begin{array}{cc}
e: a ; b ; & u, u \\
c, d:-;-; &
\end{array}\right]={ }_{2} F_{2}\left[\begin{array}{cc}
a+b, e ; & u \\
c, d ; &
\end{array}\right] .
$$

If we start with the transformation [14, Eq. (11)] with $x \rightarrow u x$,

$$
\Phi_{2}(a, a ; c ; u x,-u x)={ }_{1} F_{2}\left(a ; \frac{c}{2}, \frac{c+1}{2} ; \frac{u^{2} x^{2}}{4}\right)
$$

we have

$$
F_{2: 0 ; 0}^{1: 1 ; 1}\left[\begin{array}{cc}
e: a ; a ; & u,-u \\
c, d:-;-; &
\end{array}\right]={ }_{3} F_{4}\left[\begin{array}{c}
a, \frac{e}{2}, \frac{e+1}{2} ; \\
\frac{c}{2}, \frac{c+1}{2}, \frac{d}{2}, \frac{d+1}{2} ;
\end{array}\right] .
$$

\section{The relations from triple hypergeometric functions $H_{A}$ and $H_{B}$}

The following functions are given by Srivastava [25-27]:

$$
\begin{aligned}
& H_{A}\left(\alpha, \beta_{1}, \beta_{2} ; \gamma_{1}, \gamma_{2} ; x, y, z\right)=\sum_{m, n, p \geq 0} \frac{(\alpha)_{m+p}\left(\beta_{1}\right)_{m+n}\left(\beta_{2}\right)_{n+p}}{\left(\gamma_{1}\right)_{m}\left(\gamma_{2}\right)_{n+p} m ! n ! p !} x^{m} y^{n} z^{p} \\
& (|x|=r<1,|y|=s<1,|z|=t<(1-r)(1-s)) ; \\
& H_{B}\left(\alpha, \beta_{1}, \beta_{2} ; \gamma_{1}, \gamma_{2}, \gamma_{3} ; x, y, z\right)=\sum_{m, n, p \geq 0} \frac{(\alpha)_{m+p}\left(\beta_{1}\right)_{m+n}\left(\beta_{2}\right)_{n+p}}{\left(\gamma_{1}\right)_{m}\left(\gamma_{2}\right)_{n}\left(\gamma_{3}\right)_{p} m ! n ! p !} x^{m} y^{n} z^{p} \\
& (|x|=r,|y|=s,|z|=t ; r+s+t+2 \sqrt{r s t}<1) .
\end{aligned}
$$

We first present the identity between $H_{A}$ and ${ }_{2} F_{1}$ [26, p.103, Eq. (5.3)]

$$
H_{A}\left(\alpha, \beta_{1}, \beta_{2} ; \gamma_{1}, \beta_{2} ; x, y, z\right)=(1-y)^{-\beta_{1}}(1-z)^{-\alpha}{ }_{2} F_{1}\left[\begin{array}{cc}
\alpha, \beta_{1} ; & \frac{x}{(1-y)(1-z)} \\
\gamma_{1} ; &
\end{array}\right] \text {. }
$$


Multiplying both sides of the above transformation by

$$
x^{e_{1}-1}(1-x)^{d_{1}-e_{1}-1} y^{e_{2}-1}(1-y)^{d_{2}+\beta_{1}-e_{2}-1} z^{e_{3}-1}(1-z)^{d_{3}+\alpha-e_{3}-1}
$$

and applying the beta integral three times with respect to $x, y, z$, we get the following transformation using the same technique in Theorems 1 and 2.

$$
\begin{aligned}
& F^{(3)}\left[\begin{array}{cc}
-:: \beta_{1} ;-; \alpha: e_{1} ; e_{2} ; e_{3} ; \\
-::-;-;-: \gamma_{1}, d_{1} ; d_{2}+\beta_{1} ; d_{3}+\alpha ; & 1,1,1
\end{array}\right] \\
& =\Gamma\left[\begin{array}{l}
d_{2}-e_{2}, d_{3}-e_{3}, d_{2}+\beta_{1}, d_{3}+\alpha \\
d_{2}, d_{3}, d_{2}+\beta_{1}-e_{2}, d_{3}+\alpha-e_{3}
\end{array}\right]{ }_{5} F_{4}\left[\begin{array}{cc}
\alpha, \beta_{1}, e_{1}, 1-d_{2}, 1-d_{3} ; & 1 \\
\gamma_{1}, d_{1}, 1+e_{2}-d_{2}, 1+e_{3}-d_{3} ; & 1
\end{array}\right] .
\end{aligned}
$$

Otherwise, if we start with transformation (5.1) with $x=y=z$,

$$
H_{A}\left(\alpha, \beta_{1}, \beta_{2} ; \gamma_{1}, \beta_{2} ; x, x, x\right)=(1-x)^{-\alpha-\beta_{1}}{ }_{2} F_{1}\left[\begin{array}{cc}
\alpha, \beta_{1} ; & \frac{x}{(1-x)^{2}}
\end{array}\right]
$$

we have another transformation

$$
\begin{aligned}
F^{(3)} & {\left[\begin{array}{cr}
e:: \beta_{1} ;-; \alpha ;-;-;-; & 1,1,1 \\
\alpha+\beta_{1}+d::-;-;-; \gamma_{1} ;-;-; &
\end{array}\right] } \\
= & \Gamma\left[\begin{array}{l}
d-e, \beta_{1}+\alpha+d \\
d, \beta_{1}+\alpha+d-e
\end{array}\right]{ }_{4} F_{3}\left[\begin{array}{cc}
\alpha, \beta_{1}, e, 1-d ; & \\
\gamma_{1}, \frac{1+e-d}{2}, 1+\frac{e-d}{2} ; & -\frac{1}{4}
\end{array}\right] .
\end{aligned}
$$

If we start with the identity between $H_{B}$ and $F_{4}$ [26, p.104, Eq. (5.6)] with $x=y=z$,

$$
H_{B}\left(\alpha, \beta_{1}, \beta_{2} ; \gamma_{1}, \beta_{2}, \beta_{2} ; x, x, x\right)=(1-x)^{-\alpha-\beta_{1}} F_{4}\left[\alpha, \beta_{1} ; \gamma_{1}, \beta_{2} ; \frac{x}{(1-x)^{2}}, \frac{x^{2}}{(1-x)^{2}}\right] \text {, }
$$

we have

$$
\begin{aligned}
F^{(3)} & {\left[\begin{array}{c}
e:: \beta_{1} ; \beta_{2} ; \alpha:-;-;-; \\
\alpha+\beta_{1}+d::-;-;-: \gamma_{1} ; \beta_{2} ; \beta_{2} ; \quad 1,1,1
\end{array}\right] } \\
= & \Gamma\left[\begin{array}{l}
d-e, \beta_{1}+\alpha+d \\
d, \beta_{1}+\alpha+d-e
\end{array}\right] \sum_{m, n \geq 0} \frac{(e)_{m+2 n}(\alpha)_{m+n}\left(\beta_{1}\right)_{m+n}(1-d)_{m}\left(-\frac{1}{4}\right)^{m}\left(\frac{1}{4}\right)^{n}}{\left(\frac{1+e-d}{2}\right)_{m+n}\left(1+\frac{e-d}{2}\right)_{m+n}\left(\gamma_{1}\right)_{m}\left(\beta_{2}\right)_{n} m ! n !} .
\end{aligned}
$$

It is interesting that the pattern of the double series on the right-hand side of the above transformation is similar to Exton's [19] triple hypergeometric series.

If we start with the relation between $H_{A}$ and $G_{2}$ [28, Eq. (8.4)] with $y \rightarrow-x$ and $z \rightarrow x$,

$$
H_{A}\left(a_{1}+a_{2}-1, b_{1}, b_{2} ; a_{1}, b_{2} ; x,-x, x\right)=(1-x)^{-a_{2}} G_{2}\left(b_{1}, a_{2} ; 1-a_{1}, 1-a_{2} ; \frac{x}{1-x}, \frac{x}{1-x}\right),
$$


we have the following transformation:

$$
\begin{aligned}
F^{(3)} & {\left[\begin{array}{c}
e:: b_{1} ;-; a_{1}+a_{2}-1:-;-;-; \\
d+a_{2}::-;-;-: a_{1} ;-;-;
\end{array} \quad 1,-1,1\right] } \\
= & \Gamma\left[\begin{array}{l}
d-e, d+a_{2} \\
d, d+a_{2}-e
\end{array}\right] \sum_{m, n \geq 0} \frac{(e)_{m+n}\left(b_{1}\right)_{m}\left(a_{2}\right)_{n}\left(1-a_{1}\right)_{n-m}\left(1-a_{2}\right)_{m-n}}{(-1)^{m+n}(1+e-d)_{m+n} m ! n !},
\end{aligned}
$$

with $e$ being a nonpositive integer.

Remark Many other transformations for the multiple hypergeometric functions can be found by applying the beta integral method, and, as examples, we just present these results. Interested readers can obtain some other transformation by using the beta integral method.

\section{Competing interests}

The authors declare that they have no competing interests.

\section{Authors' contributions}

All authors contributed equally to this paper. They read and approved the final paper.

\section{Author details}

'Department of Information Technology, Hainan Medical College, Haikou, 571199, P.R. China. ${ }^{2}$ Department of Mathematics, Shanghai University, Shanghai, 200444, P.R. China.

\section{Acknowledgements}

This work is supported by the National Natural Science Foundation of China (11201291 and 11301120), the Natural Science Foundation of Shanghai (12ZR1443800) and a grant of 'The First-class Discipline of Universities in Shanghai'.

Received: 29 September 2013 Accepted: 12 November 2013 Published: 12 Dec 2013

\section{References}

1. Rainville, ED: Special Functions. Macmillan Co., New York (1960). Reprinted by Chelsea, New York (1971)

2. Bailey, WN: Generalized Hypergeometric Series. Cambridge Tracts in Mathematics and Mathematical Physics, vol. 32. Cambridge University Press, Cambridge (1935). Reprinted by Stechert-Hafner, New York (1964)

3. Burchnall, JL, Chaundy, TW: Expansions of Appell's double hypergeometric functions. II. Q. J. Math. 12(1), 112-128 (1941)

4. Srivastava, HM, Panda, R: An integral representation for the product of two Jacobi polynomials. J. Lond. Math. Soc 12(2), 419-425 (1976)

5. Buschman, RG, Srivastava, HM: Some identities and reducibility of Kampé de Fériet functions. Math. Proc. Camb. Philos. Soc. 91, 435-440 (1982)

6. Exton, H: On the reducibility of the Kampé de Fériet function. J. Comput. Appl. Math. 83, 119-121 (1997)

7. Karlsson, PW: Some reduction formulae for power series and Kampé de Fériet function. Indag. Math. 87, 31-36 (1984)

8. Krupnikov, ED: A Register of Computer-Oriented Reduction Identities for the Kampé de Fériet Function. Novosibirsk (1996)

9. Vidūnas, R: A generalization of Kummer identity. Rocky Mt. J. Math. 32(2), 919-936 (2002)

10. Srivastava, HM, Karlsson, PW: Multiple Gaussian Hypergeometric Series. Horwood, Chichester (1985)

11. Krattenthaler, C, Srinivasa Rao, K: Automatic generation of hypergeometric identities by the beta integral method. J. Comput. Appl. Math. 160, 159-173 (2003)

12. Choi, J, Rathie, AK, Srivastava, HM: Certain hypergeometric identities deducible by using the beta integral method. Bull. Korean Math. Soc. 50, 1673-1681 (2013)

13. Erdélyi, A et al. (eds.) Higher Transcendental Functions, vol. 1. McGraw-Hill, New York (1953)

14. Manako, V: A connection formula between double hypergeometric series $\Psi_{2}$ and $\Phi_{3}$. Integral Transforms Spec. Funct. 23(7), 503-508 (2012)

15. Slater, LJ: Generalized Hypergeometric Functions. Cambridge University Press, Cambridge (1966)

16. Vidūnas, R: Specialization of Appell's functions to univariate hypergeometric functions. J. Math. Anal. Appl. 355, 145-163 (2009)

17. Appell, P: Sur les fonctions hypergéométriques de plusieurs variables. Mémoir. Sci. Math. Gauthier-Villars, Paris (1925)

18. Appell, $P$, Kampé de Fériet, J: Fonctions hypergéométriques et hypersphériques Polynomes d'Hermite. Gauthier-Villars, Paris (1926)

19. Exton, H: Hypergeometric functions of three variables. J. Indian Acad. Math. 4(2), 113-119 (1982)

20. Hasanov, A, Turaev, M: Decomposition formulas for the double hypergeometric functions $G_{1}$ and $G_{2}$. Appl. Math Comput. 187, 195-201 (2007) 
21. Erdélyi, A: Hypergeometric functions of two variables. Acta Math. 83, 131-164 (1950)

22. Brychkov, YA: Handbook of Special Functions: Derivatives, Integrals, Series and Other Formulas. CRC Press, Boca Raton (2008)

23. Gradshteyn, IS, Ryzhyk, IM: Table of Integrals, Series, and Products. Academic Press, New York (2007)

24. Prudnikov, AP, Brychkov, YA, Marichev, Ol: Integrals and Series, More Special Functions, vol. 3. Gordon \& Breach, New York (1990)

25. Exton, H: On Srivastava's symmetrical triple hypergeometric function $H_{B}$. J. Indian Acad. Math. 25, 17-22 (2003)

26. Srivastava, HM: Hypergeometric functions of three variables. Ganita 15, 97-108 (1964)

27. Srivastava, HM, Monacha, HL: A Treatise on Generating Functions. Horwood, Chichester (1984)

28. Srivastava, HM: Some integrals representing triple hypergeometric series. Rend. Circ. Mat. Palermo 16(1), 99-115 (1967)

10.1186/1687-1847-2013-360

Cite this article as: Wei et al.: Certain transformations for multiple hypergeometric functions. Advances in Difference Equations 2013, 2013:360 